Acta Crystallographica Section E

Structure Reports

Online

ISSN 1600-5368

\section{4-Amino-N-(6-chloro-5-methoxy- pyrimidin-4-yl)benzenesulfonamide}

\author{
Hoong-Kun Fun, ${ }^{a *} \ddagger$ Jia Hao Goh, ${ }^{a} \S$ C. S. Chidan Kumar, ${ }^{b}$ \\ H. S. Yathirajan ${ }^{b}$ and B. Narayana ${ }^{c}$

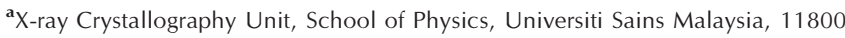 \\ USM, Penang, Malaysia, 'bepartment of Studies in Chemistry, University of Mysore, \\ Manasagangotri, Mysore 570 006, India, and ${ }^{\mathbf{C}}$ Department of Studies in Chemistry, \\ Mangalore University, Mangalagangotri, Mangalore 574 199, India \\ Correspondence e-mail: hkfun@usm.my
}

Received 5 January 2010; accepted 9 January 2010

Key indicators: single-crystal X-ray study; $T=100 \mathrm{~K}$; mean $\sigma(\mathrm{C}-\mathrm{C})=0.001 \AA$; $R$ factor $=0.030 ; w R$ factor $=0.089 ;$ data-to-parameter ratio $=21.6$.

In the title compound, $\mathrm{C}_{11} \mathrm{H}_{11} \mathrm{ClN}_{4} \mathrm{O}_{3} \mathrm{~S}$, the $\mathrm{S}$ atom is bonded in a distorted tetrahedral geometry, by two $\mathrm{O}$ atoms, a $\mathrm{C}$ atom of the benzene ring and an amino $\mathrm{N}$ atom. The essentially planar pyrimidine ring [maximum deviation $=0.020$ (1) $\AA$ ] forms a dihedral angle of $87.57(5)^{\circ}$ with the benzene ring. In the crystal structure, pairs of molecules are linked by intermolecular $\mathrm{N}-\mathrm{H} \cdots \mathrm{O}$ hydrogen bonds to generate centrosymmetric $R_{2}^{2}(8)$ ring motifs. In addition, molecules are linked into a three-dimensional extended network by intermolecular $\mathrm{N}-\mathrm{H} \cdots \mathrm{N}, \quad \mathrm{N}-\mathrm{H} \cdots \mathrm{O}$ and $\mathrm{C}-\mathrm{H} \cdots \mathrm{O}$ hydrogen bonds.

\section{Related literature}

For general background to and applications of the title compound, see: Amir et al. (2007); Calabresi et al. (1975); ElHashash et al. (1993); Nagaraja et al. (2003); Townsend \& Drach (2002). For a related structure, see: Chohan et al. (2008). For details of hydrogen-bond motifs, see: Bernstein et al. (1995). For the stability of the temperature controller used for the data collection, see: Cosier \& Glazer (1986). For bondlength data, see: Allen et al. (1987).

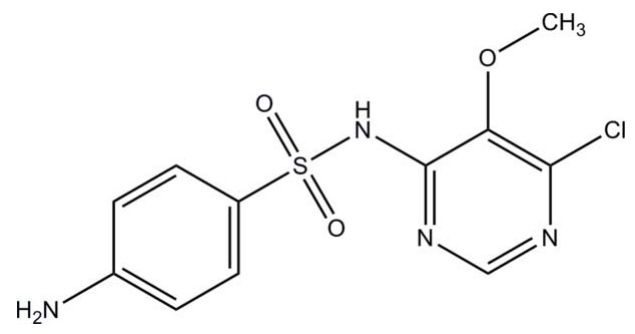

\$ Thomson Reuters ResearcherID: A-3561-2009. $\S$ Thomson Reuters ResearcherID: C-7576-2009.

\section{Experimental}

Crystal data

$$
\begin{aligned}
& \mathrm{C}_{11} \mathrm{H}_{11} \mathrm{ClN}_{4} \mathrm{O}_{3} \mathrm{~S} \\
& M_{r}=314.75 \\
& \text { Monoclinic, } P 2_{1} / c \\
& a=12.8792(6) \AA \\
& b=13.3557(6) \AA \\
& c=8.0867(4) \AA \\
& \beta=102.396(1)^{\circ}
\end{aligned}
$$

$$
\begin{aligned}
& V=1358.57(11) \AA^{3} \\
& Z=4 \\
& \text { Mo } K \alpha \text { radiation } \\
& \mu=0.45 \mathrm{~mm}^{-1} \\
& T=100 \mathrm{~K} \\
& 0.44 \times 0.33 \times 0.12 \mathrm{~mm}
\end{aligned}
$$

\section{Data collection}

Bruker SMART APEX DUO areadetector diffractometer

Absorption correction: multi-scan (SADABS; Bruker, 2009)

$T_{\min }=0.829, T_{\max }=0.950$

20122 measured reflections 4863 independent reflections 4292 reflections with $I>2 \sigma(I)$ $R_{\text {int }}=0.026$

\section{Refinement}

$R\left[F^{2}>2 \sigma\left(F^{2}\right)\right]=0.030$

$w R\left(F^{2}\right)=0.089$

$S=1.04$

4863 reflections

225 parameters

All $\mathrm{H}$-atom parameters refined

$\Delta \rho_{\max }=0.53{\mathrm{e} \AA^{-3}}^{-3}$

$\Delta \rho_{\min }=-0.37 \mathrm{e}^{-3}$

Table 1

Hydrogen-bond geometry $\left(\AA{ }^{\circ}\right)$.

\begin{tabular}{lllll}
\hline$D-\mathrm{H} \cdots A$ & $D-\mathrm{H}$ & $\mathrm{H} \cdots A$ & $D \cdots A$ & $D-\mathrm{H} \cdots A$ \\
\hline $\mathrm{N} 1-\mathrm{H} 1 N 1 \cdots \mathrm{N} 4{ }^{\mathrm{i}}$ & $0.875(19)$ & $2.616(18)$ & $3.4230(14)$ & $153.8(15)$ \\
$\mathrm{N} 1-\mathrm{H} 2 N 1 \cdots \mathrm{O} 1^{\text {ii }}$ & $0.882(18)$ & $2.533(19)$ & $3.3274(13)$ & $150.2(15)$ \\
$\mathrm{N} 2-\mathrm{H} 1 N 2 \cdots \mathrm{O} 2^{\text {ii }}$ & $0.880(18)$ & $2.031(18)$ & $2.8866(12)$ & $163.7(16)$ \\
$\mathrm{C} 4-\mathrm{H} 4 A \cdots \mathrm{O} 1^{\mathrm{ii}}$ & $0.944(16)$ & $2.460(16)$ & $3.2603(13)$ & $142.5(13)$ \\
\hline
\end{tabular}

Symmetry codes: (i) $-x+1, y+\frac{1}{2},-z+\frac{5}{2}$; (ii) $x, y, z+1$; (iii) $-x,-y+1,-z+2$.

Data collection: APEX2 (Bruker, 2009); cell refinement: SAINT (Bruker, 2009); data reduction: $S A I N T$; $\operatorname{program}(\mathrm{s})$ used to solve structure: SHELXTL (Sheldrick, 2008); program(s) used to refine structure: SHELXTL; molecular graphics: SHELXTL; software used to prepare material for publication: SHELXTL and PLATON (Spek, 2009).

HKF and JHG thank Universiti Sains Malaysia (USM) for the Research University Golden Goose grant (No. 1001/ PFIZIK/811012). JHG also thanks USM for the award of a USM fellowship. CSC thanks the University of Mysore for research facilities.

Supplementary data and figures for this paper are available from the IUCr electronic archives (Reference: LH2975).

\title{
References
}

Allen, F. H., Kennard, O., Watson, D. G., Brammer, L., Orpen, A. G. \& Taylor, R. (1987). J. Chem. Soc. Perkin Trans. 2, pp. S1-19.

Amir, M., Javed, S. A. \& Kumar, H. (2007). Indian J. Pharm. Sci. 69, 337-343. Bernstein, J., Davis, R. E., Shimoni, L. \& Chang, N.-L. (1995). Angew. Chem. Int. Ed. Engl. 34, 1555-1573.

Bruker (2009). APEX2, SAINT and SADABS. Bruker AXS Inc., Madison, Wisconsin, USA.

Calabresi, P., Parks, R. E., Goodman, L. S. \& Gilman, A. (1975). The Pharmacological Basis of Therapeutics, 5th ed., p. 1254. New York: Macmillan.

Chohan, Z. H., Tahir, M. N., Shad, H. A. \& Khan, I. U. (2008). Acta Cryst. E64 0648 .

Cosier, J. \& Glazer, A. M. (1986). J. Appl. Cryst. 19, 105-107. 


\section{organic compounds}

El-Hashash, M. A., Mahmoud, M. R. \& Madboli, S. A. (1993). Indian J. Chem. Sect B, 32, 449-451.

Nagaraja, P., Yathirajan, H. S., Raju, C. R., Vasantha, R. A., Nagendra, P. \& Hemantha Kumar, M. S. (2003). Il Farmaco, 58, 1295-1300.
Sheldrick, G. M. (2008). Acta Cryst. A64, 112-122.

Spek, A. L. (2009). Acta Cryst. D65, 148-155.

Townsend, L. B. \& Drach, J. C. (2002). Chem. Abstr. 136, 134778. 


\title{
supporting information
}

Acta Cryst. (2010). E66, o372-0373 [https://doi.org/10.1107/S1600536810001121]

\section{4-Amino-N-(6-chloro-5-methoxypyrimidin-4-yl) benzenesulfonamide}

\author{
Hoong-Kun Fun, Jia Hao Goh, C. S. Chidan Kumar, H. S. Yathirajan and B. Narayana
}

\section{S1. Comment}

The importance of pyrimidines and analogous compounds in pharmaceutical and biological fields is well known (Townsend et al., 2002). Some substituted pyrimidines and their derivaties have been reported to possess anti-microbial and anti-fungal activities (El-Hashash et al., 1993). Pyrimidines have incidental anti-viral activity against herpes and vaccinia infections (Calabresi et al., 1975). A review on pyrimidines as anti-inflammatory agent is described by Amir et al. (2007). Sulfonamides are an important class of anti-bacterial drugs used in medicine and veterinary practice. Sulfa drugs are widely used in the treatment of infections, especially for patients intolerant to antibiotics. The vast commercial success of these medicinal agents has made the chemistry of sulfonamides to become a major area of research and an important branch of commercial importance in pharmaceutical sciences (Nagaraja et al., 2003). In view of the importance of the title compound possessing potential anti-bacterial properties, its crystal structure is reported herein.

In the title sulfonamide compound (Fig. 1), the geometry around the S1 atom is a distorted tetrahedron, comprising of atoms $\mathrm{O} 1$ and $\mathrm{O} 2$ of the sulfonyl group, $\mathrm{C} 6$ atom of benzene ring and the amino atom N2. The O1-S1-O2 and O2-S1$\mathrm{N} 2$ angles are $119.20(5)$ and $102.20(4)^{\circ}$, respectively, and the C6-S1-N2-C7 torsion angle is $-68.95(9)^{\circ}$. The pyrimidine ring is essentially planar, with r.m.s. deviation of -0.020 (1) $\AA$, and is almost perpendicular to the benzene ring (C1-C6), as indicated by the dihedral angle of $87.57(5)^{\circ}$. The bond lengths (Allen et al., 1987) and angles are within normal ranges and are comparable to a related structure (Chohan et al., 2008). In the crystal structure, pairs of intermolecular $\mathrm{N} 2-\mathrm{H} 1 \mathrm{~N} 2 \cdots \mathrm{O} 2^{\text {iii }}$ hydrogen bonds (see Table 1 for symmetry code) generate $R^{2}{ }_{2}(8)$ ring motifs (Bernstein et al., 1995). Neighbouring molecules are linked into a three-dimensional extended network by intermolecular N1$\mathrm{H} 1 \mathrm{~N} 1 \cdots \mathrm{N} 4, \mathrm{~N} 1-\mathrm{H} 2 \mathrm{~N} 1 \cdots \mathrm{O} 1$ and $\mathrm{C} 4-\mathrm{H} 4 \mathrm{~A} \cdots \mathrm{O} 1$ hydrogen bonds (Fig. 2).

\section{S2. Experimental}

The title compound was obtained as a gift sample from R. L. Fine Chem, Bangalore, India. The compound was used without further purification. Single crystals of good quality were obtained from slow evaporation of an acetonitrile solution. M.p. 447-450 K.

\section{S3. Refinement}

All the $\mathrm{H}$ atoms were located in a difference Fourier map and allowed to refine freely [range of $\mathrm{C}-\mathrm{H}=0.90$ (2) -0.991 (19) $\AA]$. 


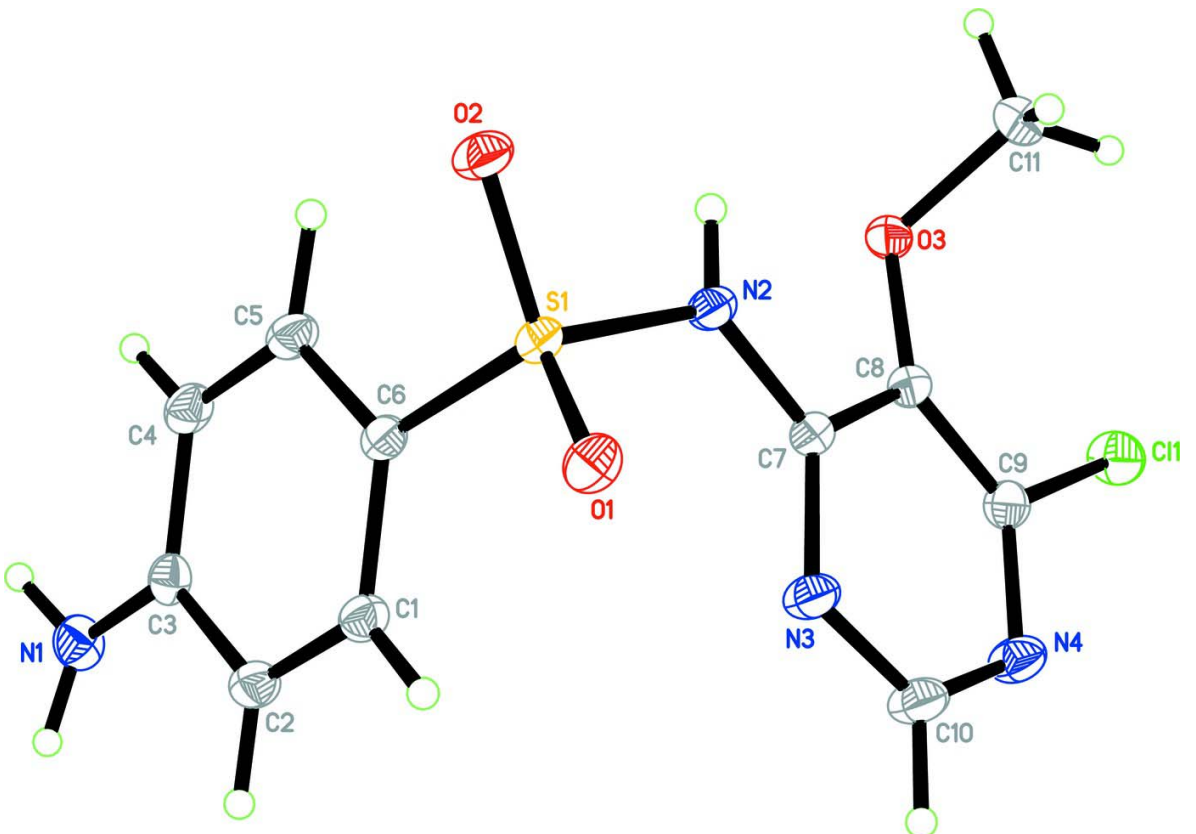

Figure 1

The molecular structure of the title compound, showing $50 \%$ probability displacement ellipsoids for non- $\mathrm{H}$ atoms and the atom-numbering scheme.

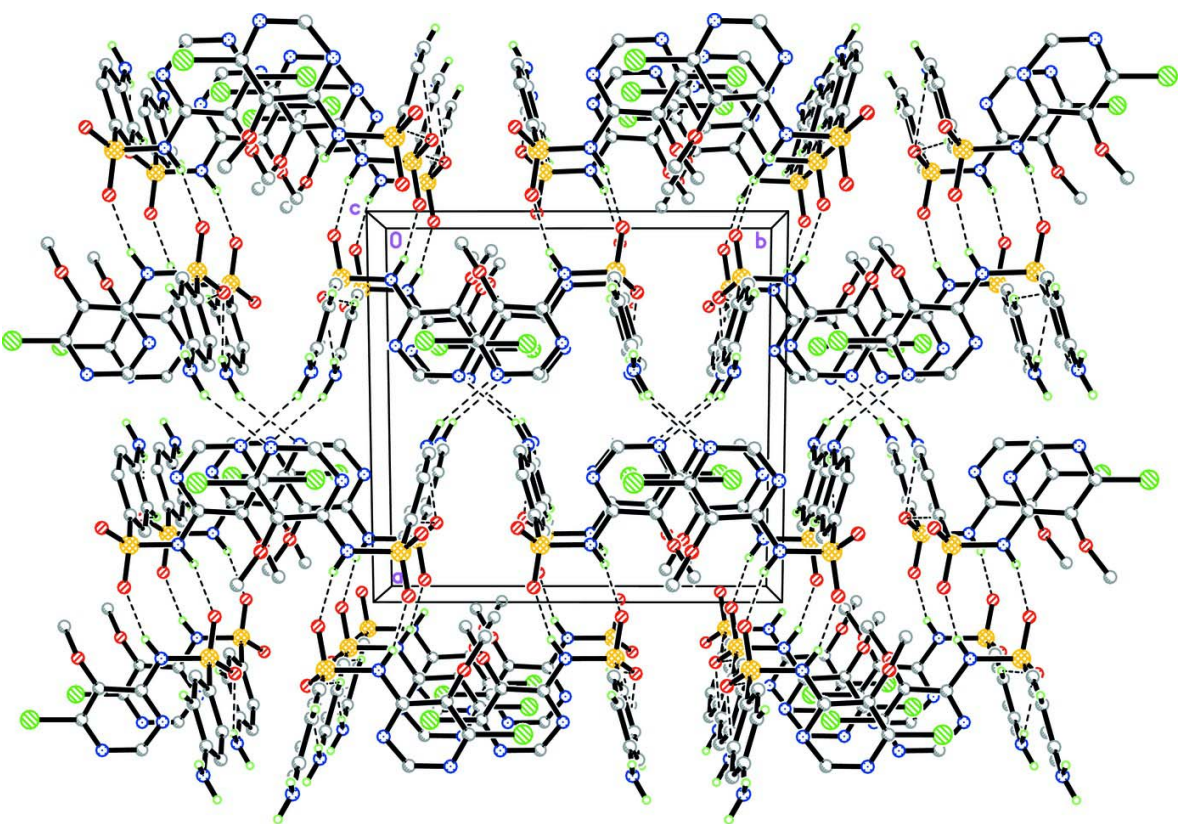

Figure 2

Part of the crystal structure of the title compound, viewed along the $c$ axis, showing a three-dimensional extended network. $\mathrm{H}$ atoms not involved in intermolecular hydrogen bonds (dashed lines) have been omitted for clarity. 
4-Amino-N-(6-chloro-5-methoxypyrimidin-4-yl)benzenesulfonamide

Crystal data

$\mathrm{C}_{11} \mathrm{H}_{11} \mathrm{ClN}_{4} \mathrm{O}_{3} \mathrm{~S}$

$M_{r}=314.75$

Monoclinic, $P 2_{1} / c$

Hall symbol: -P $2 \mathrm{ybc}$

$a=12.8792(6) \AA$

$b=13.3557(6) \AA$

$c=8.0867(4) \AA$

$\beta=102.396(1)^{\circ}$

$V=1358.57(11) \AA^{3}$

$Z=4$

\section{Data collection}

Bruker SMART APEX DUO area-detector diffractometer

Radiation source: fine-focus sealed tube

Graphite monochromator

$\varphi$ and $\omega$ scans

Absorption correction: multi-scan

(SADABS; Bruker, 2009)

$T_{\text {min }}=0.829, T_{\max }=0.950$

\section{Refinement}

Refinement on $F^{2}$

Least-squares matrix: full

$R\left[F^{2}>2 \sigma\left(F^{2}\right)\right]=0.030$

$w R\left(F^{2}\right)=0.089$

$S=1.04$

4863 reflections

225 parameters

0 restraints

Primary atom site location: structure-invariant direct methods
$F(000)=648$

$D_{\mathrm{x}}=1.539 \mathrm{Mg} \mathrm{m}^{-3}$

Mo $K \alpha$ radiation, $\lambda=0.71073 \AA$

Cell parameters from 9451 reflections

$\theta=3.1-35.0^{\circ}$

$\mu=0.45 \mathrm{~mm}^{-1}$

$T=100 \mathrm{~K}$

Plate, colourless

$0.44 \times 0.33 \times 0.12 \mathrm{~mm}$

20122 measured reflections

4863 independent reflections

4292 reflections with $I>2 \sigma(I)$

$R_{\text {int }}=0.026$

$\theta_{\text {max }}=32.5^{\circ}, \theta_{\min }=1.6^{\circ}$

$h=-19 \rightarrow 18$

$k=-20 \rightarrow 20$

$l=-11 \rightarrow 12$

Secondary atom site location: difference Fourier map

Hydrogen site location: inferred from

neighbouring sites

All H-atom parameters refined

$w=1 /\left[\sigma^{2}\left(F_{\mathrm{o}}^{2}\right)+(0.0475 P)^{2}+0.4528 P\right]$

where $P=\left(F_{\mathrm{o}}^{2}+2 F_{\mathrm{c}}^{2}\right) / 3$

$(\Delta / \sigma)_{\max }<0.001$

$\Delta \rho_{\max }=0.53$ e $\AA^{-3}$

$\Delta \rho_{\min }=-0.37$ e $\AA^{-3}$

Special details

Experimental. The crystal was placed in the cold stream of an Oxford Cyrosystems Cobra open-flow nitrogen cryostat (Cosier \& Glazer, 1986) operating at 100.0 (1)K.

Geometry. All esds (except the esd in the dihedral angle between two 1.s. planes) are estimated using the full covariance matrix. The cell esds are taken into account individually in the estimation of esds in distances, angles and torsion angles; correlations between esds in cell parameters are only used when they are defined by crystal symmetry. An approximate (isotropic) treatment of cell esds is used for estimating esds involving l.s. planes.

Refinement. Refinement of $\mathrm{F}^{2}$ against ALL reflections. The weighted R-factor wR and goodness of fit $\mathrm{S}$ are based on $\mathrm{F}^{2}$, conventional R-factors $\mathrm{R}$ are based on $\mathrm{F}$, with $\mathrm{F}$ set to zero for negative $\mathrm{F}^{2}$. The threshold expression of $\mathrm{F}^{2}>2 \operatorname{sigma}\left(\mathrm{F}^{2}\right)$ is used only for calculating R-factors(gt) etc. and is not relevant to the choice of reflections for refinement. R-factors based on $\mathrm{F}^{2}$ are statistically about twice as large as those based on F, and R- factors based on ALL data will be even larger.

Fractional atomic coordinates and isotropic or equivalent isotropic displacement parameters $\left(\AA^{2}\right)$

\begin{tabular}{lllll}
\hline & $x$ & $y$ & $z$ & $U_{\text {iso }} * / U_{\text {eq }}$ \\
\hline C11 & $0.32221(2)$ & $0.130087(19)$ & $0.89383(4)$ & $0.02300(7)$ \\
S1 & $0.150384(18)$ & $0.591224(17)$ & $0.98106(3)$ & $0.01312(6)$
\end{tabular}




$\begin{array}{lllll}\mathrm{O} 1 & 0.19845(6) & 0.64388(6) & 0.86316(9) & 0.01714(14) \\ \mathrm{O} 2 & 0.03978(6) & 0.60773(6) & 0.97922(10) & 0.01793(14) \\ \mathrm{O} 3 & 0.15020(6) & 0.26617(6) & 0.97198(9) & 0.01711(14) \\ \mathrm{N} 1 & 0.40417(8) & 0.62924(7) & 1.67597(11) & 0.02030(18) \\ \mathrm{N} 2 & 0.15327(7) & 0.46898(6) & 0.94120(11) & 0.01577(15) \\ \mathrm{N} 3 & 0.32756(7) & 0.46232(7) & 0.89578(12) & 0.01792(16) \\ \mathrm{N} 4 & 0.40819(7) & 0.30443(7) & 0.86117(13) & 0.01997(17) \\ \mathrm{C} 1 & 0.32974(8) & 0.63720(7) & 1.21150(12) & 0.01551(17) \\ \mathrm{C} 2 & 0.38928(8) & 0.64543(8) & 1.37493(13) & 0.01691(17) \\ \mathrm{C} 3 & 0.34401(8) & 0.62413(7) & 1.51452(12) & 0.01546(17) \\ \mathrm{C} 4 & 0.23638(8) & 0.59518(8) & 1.48550(13) & 0.01756(18) \\ \mathrm{C} 5 & 0.17708(8) & 0.58676(8) & 1.32262(13) & 0.01685(18) \\ \mathrm{C} 6 & 0.22376(7) & 0.60750(7) & 1.18497(12) & 0.01355(16) \\ \mathrm{C} 7 & 0.24116(7) & 0.41496(7) & 0.92115(12) & 0.01407(16) \\ \mathrm{C} 8 & 0.23504(7) & 0.30973(7) & 0.92579(12) & 0.01413(16) \\ \mathrm{C} 9 & 0.32214(8) & 0.25928(7) & 0.89322(12) & 0.01631(17) \\ \mathrm{C} 10 & 0.40614(8) & 0.40413(8) & 0.86664(15) & 0.0207(2) \\ \mathrm{C} 11 & 0.07329(9) & 0.21978(10) & 0.83669(16) & 0.0253(2) \\ \mathrm{H} 1 \mathrm{~A} & 0.3591(13) & 0.6546(12) & 1.120(2) & 0.025(4)^{*} \\ \mathrm{H} 2 \mathrm{~A} & 0.4615(13) & 0.6672(13) & 1.393(2) & 0.027(4)^{*} \\ \mathrm{H} 4 \mathrm{~A} & 0.2078(13) & 0.5806(12) & 1.581(2) & 0.027(4)^{*} \\ \mathrm{H} 5 \mathrm{~A} & 0.1038(13) & 0.5624(12) & 1.303(2) & 0.026(4)^{*} \\ \mathrm{H} 10 \mathrm{~A} & 0.4693(14) & 0.4379(13) & 0.847(2) & 0.030(4)^{*} \\ \mathrm{H} 11 \mathrm{~A} & 0.0146(14) & 0.2042(14) & 0.883(2) & 0.035(4)^{*} \\ \mathrm{H} 11 \mathrm{~B} & 0.0532(14) & 0.2688(14) & 0.743(2) & 0.038(5)^{*} \\ \mathrm{H} 11 \mathrm{C} & 0.0991(15) & 0.1654(16) & 0.795(2) & 0.043(5)^{*} \\ \mathrm{H} 1 \mathrm{~N} 1 & 0.4646(14) & 0.6617(14) & 1.695(2) & 0.033(4)^{*} \\ \mathrm{H} 2 \mathrm{~N} 1 & 0.3696(15) & 0.6271(14) & 1.759(2) & 0.034(5)^{*} \\ \mathrm{H} 1 \mathrm{~N} 2 & 0.1012(14) & 0.4342(13) & 0.968(2) & 0.030(4)^{*}\end{array}$

Atomic displacement parameters $\left(\AA^{2}\right)$

\begin{tabular}{lllllll}
\hline & $U^{11}$ & $U^{22}$ & $U^{33}$ & $U^{12}$ & $U^{13}$ & $U^{23}$ \\
\hline C11 & $0.02251(13)$ & $0.01320(11)$ & $0.03324(15)$ & $0.00115(8)$ & $0.00591(10)$ & $-0.00126(9)$ \\
S1 & $0.01184(10)$ & $0.01368(11)$ & $0.01457(11)$ & $0.00149(7)$ & $0.00442(8)$ & $0.00027(7)$ \\
O1 & $0.0191(3)$ & $0.0179(3)$ & $0.0156(3)$ & $0.0009(3)$ & $0.0064(3)$ & $0.0026(2)$ \\
O2 & $0.0121(3)$ & $0.0195(3)$ & $0.0228(3)$ & $0.0032(2)$ & $0.0050(3)$ & $0.0005(3)$ \\
O3 & $0.0161(3)$ & $0.0188(3)$ & $0.0177(3)$ & $-0.0056(3)$ & $0.0064(3)$ & $-0.0027(3)$ \\
N1 & $0.0241(4)$ & $0.0228(4)$ & $0.0140(4)$ & $-0.0020(3)$ & $0.0041(3)$ & $-0.0002(3)$ \\
N2 & $0.0129(3)$ & $0.0139(3)$ & $0.0220(4)$ & $-0.0005(3)$ & $0.0071(3)$ & $-0.0028(3)$ \\
N3 & $0.0143(4)$ & $0.0154(4)$ & $0.0260(4)$ & $0.0000(3)$ & $0.0085(3)$ & $-0.0005(3)$ \\
N4 & $0.0161(4)$ & $0.0172(4)$ & $0.0285(4)$ & $0.0019(3)$ & $0.0091(3)$ & $0.0003(3)$ \\
C1 & $0.0155(4)$ & $0.0164(4)$ & $0.0160(4)$ & $-0.0008(3)$ & $0.0063(3)$ & $0.0003(3)$ \\
C2 & $0.0159(4)$ & $0.0189(4)$ & $0.0167(4)$ & $-0.0015(3)$ & $0.0053(3)$ & $0.0000(3)$ \\
C3 & $0.0191(4)$ & $0.0130(4)$ & $0.0148(4)$ & $0.0011(3)$ & $0.0049(3)$ & $-0.0002(3)$ \\
C4 & $0.0202(4)$ & $0.0185(4)$ & $0.0161(4)$ & $-0.0004(3)$ & $0.0087(3)$ & $0.0009(3)$ \\
C5 & $0.0157(4)$ & $0.0188(4)$ & $0.0178(4)$ & $-0.0007(3)$ & $0.0076(3)$ & $0.0008(3)$ \\
C6 & $0.0135(4)$ & $0.0136(4)$ & $0.0145(4)$ & $0.0006(3)$ & $0.0049(3)$ & $0.0000(3)$
\end{tabular}




\begin{tabular}{lllllll} 
C7 & $0.0123(4)$ & $0.0149(4)$ & $0.0154(4)$ & $0.0003(3)$ & $0.0041(3)$ & $-0.0017(3)$ \\
C8 & $0.0137(4)$ & $0.0148(4)$ & $0.0144(4)$ & $-0.0013(3)$ & $0.0041(3)$ & $-0.0010(3)$ \\
C9 & $0.0167(4)$ & $0.0135(4)$ & $0.0189(4)$ & $0.0011(3)$ & $0.0042(3)$ & $-0.0011(3)$ \\
C10 & $0.0153(4)$ & $0.0180(4)$ & $0.0313(5)$ & $0.0004(3)$ & $0.0103(4)$ & $-0.0002(4)$ \\
C11 & $0.0196(5)$ & $0.0299(6)$ & $0.0268(5)$ & $-0.0086(4)$ & $0.0058(4)$ & $-0.0109(4)$ \\
\hline
\end{tabular}

Geometric parameters $\left(\hat{A},{ }^{\circ}\right)$

\begin{tabular}{|c|c|c|c|}
\hline $\mathrm{C} 11-\mathrm{C} 9$ & $1.7255(10)$ & $\mathrm{C} 1-\mathrm{C} 2$ & $1.3826(14)$ \\
\hline $\mathrm{S} 1-\mathrm{O} 1$ & $1.4283(7)$ & $\mathrm{C} 1-\mathrm{C} 6$ & $1.3932(13)$ \\
\hline $\mathrm{S} 1-\mathrm{O} 2$ & $1.4383(7)$ & $\mathrm{C} 1-\mathrm{H} 1 \mathrm{~A}$ & $0.931(16)$ \\
\hline $\mathrm{S} 1-\mathrm{N} 2$ & $1.6662(9)$ & $\mathrm{C} 2-\mathrm{C} 3$ & $1.4063(13)$ \\
\hline S1-C6 & $1.7292(10)$ & $\mathrm{C} 2-\mathrm{H} 2 \mathrm{~A}$ & $0.955(17)$ \\
\hline $\mathrm{O} 3-\mathrm{C} 8$ & $1.3590(11)$ & $\mathrm{C} 3-\mathrm{C} 4$ & $1.4094(14)$ \\
\hline $\mathrm{O} 3-\mathrm{C} 11$ & $1.4480(13)$ & $\mathrm{C} 4-\mathrm{C} 5$ & $1.3781(15)$ \\
\hline $\mathrm{N} 1-\mathrm{C} 3$ & $1.3694(13)$ & $\mathrm{C} 4-\mathrm{H} 4 \mathrm{~A}$ & $0.941(17)$ \\
\hline $\mathrm{N} 1-\mathrm{H} 1 \mathrm{~N} 1$ & $0.875(18)$ & $\mathrm{C} 5-\mathrm{C} 6$ & $1.4016(13)$ \\
\hline $\mathrm{N} 1-\mathrm{H} 2 \mathrm{~N} 1$ & $0.879(19)$ & $\mathrm{C} 5-\mathrm{H} 5 \mathrm{~A}$ & $0.979(16)$ \\
\hline $\mathrm{N} 2-\mathrm{C} 7$ & $1.3809(12)$ & $\mathrm{C} 7-\mathrm{C} 8$ & $1.4085(14)$ \\
\hline $\mathrm{N} 2-\mathrm{H} 1 \mathrm{~N} 2$ & $0.880(18)$ & $\mathrm{C} 8-\mathrm{C} 9$ & $1.3816(13)$ \\
\hline N3-C7 & $1.3336(12)$ & $\mathrm{C} 10-\mathrm{H} 10 \mathrm{~A}$ & $0.973(17)$ \\
\hline $\mathrm{N} 3-\mathrm{C} 10$ & $1.3364(13)$ & $\mathrm{C} 11-\mathrm{H} 11 \mathrm{~A}$ & $0.938(18)$ \\
\hline $\mathrm{N} 4-\mathrm{C} 10$ & $1.3328(14)$ & $\mathrm{C} 11-\mathrm{H} 11 \mathrm{~B}$ & $0.991(19)$ \\
\hline $\mathrm{N} 4-\mathrm{C} 9$ & $1.3351(13)$ & $\mathrm{C} 11-\mathrm{H} 11 \mathrm{C}$ & $0.90(2)$ \\
\hline $\mathrm{O} 1-\mathrm{S} 1-\mathrm{O} 2$ & $119.20(5)$ & $\mathrm{C} 3-\mathrm{C} 4-\mathrm{H} 4 \mathrm{~A}$ & $117.7(10)$ \\
\hline $\mathrm{O} 1-\mathrm{S} 1-\mathrm{N} 2$ & $108.81(4)$ & $\mathrm{C} 4-\mathrm{C} 5-\mathrm{C} 6$ & $119.92(9)$ \\
\hline $\mathrm{O} 2-\mathrm{S} 1-\mathrm{N} 2$ & $102.20(4)$ & $\mathrm{C} 4-\mathrm{C} 5-\mathrm{H} 5 \mathrm{~A}$ & $119.9(10)$ \\
\hline $\mathrm{O} 1-\mathrm{S} 1-\mathrm{C} 6$ & $110.38(5)$ & $\mathrm{C} 6-\mathrm{C} 5-\mathrm{H} 5 \mathrm{~A}$ & $120.1(10)$ \\
\hline $\mathrm{O} 2-\mathrm{S} 1-\mathrm{C} 6$ & $109.14(5)$ & $\mathrm{C} 1-\mathrm{C} 6-\mathrm{C} 5$ & $120.47(9)$ \\
\hline $\mathrm{N} 2-\mathrm{S} 1-\mathrm{C} 6$ & $106.10(5)$ & $\mathrm{C} 1-\mathrm{C} 6-\mathrm{S} 1$ & $119.96(7)$ \\
\hline $\mathrm{C} 8-\mathrm{O} 3-\mathrm{C} 11$ & $115.81(8)$ & $\mathrm{C} 5-\mathrm{C} 6-\mathrm{S} 1$ & $119.52(8)$ \\
\hline $\mathrm{C} 3-\mathrm{N} 1-\mathrm{H} 1 \mathrm{~N} 1$ & $119.3(12)$ & $\mathrm{N} 3-\mathrm{C} 7-\mathrm{N} 2$ & $120.16(9)$ \\
\hline $\mathrm{C} 3-\mathrm{N} 1-\mathrm{H} 2 \mathrm{~N} 1$ & $116.6(12)$ & $\mathrm{N} 3-\mathrm{C} 7-\mathrm{C} 8$ & $122.05(9)$ \\
\hline $\mathrm{H} 1 \mathrm{~N} 1-\mathrm{N} 1-\mathrm{H} 2 \mathrm{~N} 1$ & $117.5(17)$ & $\mathrm{N} 2-\mathrm{C} 7-\mathrm{C} 8$ & $117.78(8)$ \\
\hline $\mathrm{C} 7-\mathrm{N} 2-\mathrm{S} 1$ & $125.98(7)$ & $\mathrm{O} 3-\mathrm{C} 8-\mathrm{C} 9$ & $125.25(9)$ \\
\hline $\mathrm{C} 7-\mathrm{N} 2-\mathrm{H} 1 \mathrm{~N} 2$ & $116.1(11)$ & $\mathrm{O} 3-\mathrm{C} 8-\mathrm{C} 7$ & $119.17(8)$ \\
\hline $\mathrm{S} 1-\mathrm{N} 2-\mathrm{H} 1 \mathrm{~N} 2$ & $114.8(11)$ & $\mathrm{C} 9-\mathrm{C} 8-\mathrm{C} 7$ & $115.41(9)$ \\
\hline $\mathrm{C} 7-\mathrm{N} 3-\mathrm{C} 10$ & $116.09(9)$ & $\mathrm{N} 4-\mathrm{C} 9-\mathrm{C} 8$ & $123.96(9)$ \\
\hline $\mathrm{C} 10-\mathrm{N} 4-\mathrm{C} 9$ & $114.94(9)$ & $\mathrm{N} 4-\mathrm{C} 9-\mathrm{Cl1}$ & $116.89(7)$ \\
\hline $\mathrm{C} 2-\mathrm{C} 1-\mathrm{C} 6$ & $119.60(9)$ & $\mathrm{C} 8-\mathrm{C} 9-\mathrm{Cl} 1$ & $119.15(8)$ \\
\hline $\mathrm{C} 2-\mathrm{C} 1-\mathrm{H} 1 \mathrm{~A}$ & $120.2(10)$ & $\mathrm{N} 4-\mathrm{C} 10-\mathrm{N} 3$ & $127.44(10)$ \\
\hline $\mathrm{C} 6-\mathrm{C} 1-\mathrm{H} 1 \mathrm{~A}$ & $120.1(10)$ & $\mathrm{N} 4-\mathrm{C} 10-\mathrm{H} 10 \mathrm{~A}$ & $115.8(10)$ \\
\hline $\mathrm{C} 1-\mathrm{C} 2-\mathrm{C} 3$ & $120.71(9)$ & $\mathrm{N} 3-\mathrm{C} 10-\mathrm{H} 10 \mathrm{~A}$ & $116.8(10)$ \\
\hline $\mathrm{C} 1-\mathrm{C} 2-\mathrm{H} 2 \mathrm{~A}$ & $119.7(10)$ & $\mathrm{O} 3-\mathrm{C} 11-\mathrm{H} 11 \mathrm{~A}$ & $105.7(11)$ \\
\hline $\mathrm{C} 3-\mathrm{C} 2-\mathrm{H} 2 \mathrm{~A}$ & $119.6(10)$ & $\mathrm{O} 3-\mathrm{C} 11-\mathrm{H} 11 \mathrm{~B}$ & $108.5(11)$ \\
\hline $\mathrm{N} 1-\mathrm{C} 3-\mathrm{C} 2$ & $120.53(9)$ & $\mathrm{H} 11 \mathrm{~A}-\mathrm{C} 11-\mathrm{H} 11 \mathrm{~B}$ & $110.6(15)$ \\
\hline $\mathrm{N} 1-\mathrm{C} 3-\mathrm{C} 4$ & $120.49(9)$ & $\mathrm{O} 3-\mathrm{C} 11-\mathrm{H} 11 \mathrm{C}$ & $112.6(12)$ \\
\hline
\end{tabular}




\begin{tabular}{|c|c|c|c|}
\hline $\mathrm{C} 2-\mathrm{C} 3-\mathrm{C} 4$ & $118.96(9)$ & $\mathrm{H} 11 \mathrm{~A}-\mathrm{C} 11-\mathrm{H} 11 \mathrm{C}$ & $111.6(17)$ \\
\hline $\mathrm{C} 5-\mathrm{C} 4-\mathrm{C} 3$ & $120.34(9)$ & $\mathrm{H} 11 \mathrm{~B}-\mathrm{C} 11-\mathrm{H} 11 \mathrm{C}$ & $107.8(16)$ \\
\hline $\mathrm{C} 5-\mathrm{C} 4-\mathrm{H} 4 \mathrm{~A}$ & $122.0(10)$ & & \\
\hline $\mathrm{O} 1-\mathrm{S} 1-\mathrm{N} 2-\mathrm{C} 7$ & $49.81(10)$ & $\mathrm{C} 10-\mathrm{N} 3-\mathrm{C} 7-\mathrm{N} 2$ & $-175.76(10)$ \\
\hline $\mathrm{O} 2-\mathrm{S} 1-\mathrm{N} 2-\mathrm{C} 7$ & $176.75(8)$ & $\mathrm{C} 10-\mathrm{N} 3-\mathrm{C} 7-\mathrm{C} 8$ & $3.19(15)$ \\
\hline $\mathrm{C} 6-\mathrm{S} 1-\mathrm{N} 2-\mathrm{C} 7$ & $-68.95(9)$ & $\mathrm{S} 1-\mathrm{N} 2-\mathrm{C} 7-\mathrm{N} 3$ & $-14.89(14)$ \\
\hline $\mathrm{C} 6-\mathrm{C} 1-\mathrm{C} 2-\mathrm{C} 3$ & $0.01(15)$ & $\mathrm{S} 1-\mathrm{N} 2-\mathrm{C} 7-\mathrm{C} 8$ & $166.11(7)$ \\
\hline $\mathrm{C} 1-\mathrm{C} 2-\mathrm{C} 3-\mathrm{N} 1$ & $177.89(10)$ & $\mathrm{C} 11-\mathrm{O} 3-\mathrm{C} 8-\mathrm{C} 9$ & $-78.05(13)$ \\
\hline $\mathrm{C} 1-\mathrm{C} 2-\mathrm{C} 3-\mathrm{C} 4$ & $-0.66(15)$ & $\mathrm{C} 11-\mathrm{O} 3-\mathrm{C} 8-\mathrm{C} 7$ & $106.84(11)$ \\
\hline $\mathrm{N} 1-\mathrm{C} 3-\mathrm{C} 4-\mathrm{C} 5$ & $-177.74(10)$ & $\mathrm{N} 3-\mathrm{C} 7-\mathrm{C} 8-\mathrm{O} 3$ & $172.23(9)$ \\
\hline $\mathrm{C} 2-\mathrm{C} 3-\mathrm{C} 4-\mathrm{C} 5$ & $0.80(15)$ & $\mathrm{N} 2-\mathrm{C} 7-\mathrm{C} 8-\mathrm{O} 3$ & $-8.80(13)$ \\
\hline $\mathrm{C} 3-\mathrm{C} 4-\mathrm{C} 5-\mathrm{C} 6$ & $-0.30(15)$ & $\mathrm{N} 3-\mathrm{C} 7-\mathrm{C} 8-\mathrm{C} 9$ & $-3.36(14)$ \\
\hline $\mathrm{C} 2-\mathrm{C} 1-\mathrm{C} 6-\mathrm{C} 5$ & $0.51(15)$ & $\mathrm{N} 2-\mathrm{C} 7-\mathrm{C} 8-\mathrm{C} 9$ & $175.62(9)$ \\
\hline $\mathrm{C} 2-\mathrm{C} 1-\mathrm{C} 6-\mathrm{S} 1$ & $-176.93(8)$ & $\mathrm{C} 10-\mathrm{N} 4-\mathrm{C} 9-\mathrm{C} 8$ & $1.60(15)$ \\
\hline $\mathrm{C} 4-\mathrm{C} 5-\mathrm{C} 6-\mathrm{C} 1$ & $-0.37(15)$ & $\mathrm{C} 10-\mathrm{N} 4-\mathrm{C} 9-\mathrm{Cl1}$ & $-178.19(8)$ \\
\hline $\mathrm{C} 4-\mathrm{C} 5-\mathrm{C} 6-\mathrm{S} 1$ & $177.08(8)$ & $\mathrm{O} 3-\mathrm{C} 8-\mathrm{C} 9-\mathrm{N} 4$ & $-174.46(10)$ \\
\hline $\mathrm{O} 1-\mathrm{S} 1-\mathrm{C} 6-\mathrm{C} 1$ & $-20.24(9)$ & $\mathrm{C} 7-\mathrm{C} 8-\mathrm{C} 9-\mathrm{N} 4$ & $0.82(14)$ \\
\hline $\mathrm{O} 2-\mathrm{S} 1-\mathrm{C} 6-\mathrm{C} 1$ & $-153.06(8)$ & $\mathrm{O} 3-\mathrm{C} 8-\mathrm{C} 9-\mathrm{Cl1}$ & $5.32(14)$ \\
\hline $\mathrm{N} 2-\mathrm{S} 1-\mathrm{C} 6-\mathrm{C} 1$ & $97.49(8)$ & $\mathrm{C} 7-\mathrm{C} 8-\mathrm{C} 9-\mathrm{Cl1}$ & $-179.40(7)$ \\
\hline $\mathrm{O} 1-\mathrm{S} 1-\mathrm{C} 6-\mathrm{C} 5$ & $162.29(8)$ & $\mathrm{C} 9-\mathrm{N} 4-\mathrm{C} 10-\mathrm{N} 3$ & $-1.89(18)$ \\
\hline $\mathrm{O} 2-\mathrm{S} 1-\mathrm{C} 6-\mathrm{C} 5$ & $29.47(9)$ & $\mathrm{C} 7-\mathrm{N} 3-\mathrm{C} 10-\mathrm{N} 4$ & $-0.47(18)$ \\
\hline $\mathrm{N} 2-\mathrm{S} 1-\mathrm{C} 6-\mathrm{C} 5$ & $-79.98(9)$ & & \\
\hline
\end{tabular}

Hydrogen-bond geometry $\left(\AA,{ }^{\circ}\right)$

\begin{tabular}{lllll}
\hline$D-\mathrm{H} \cdots A$ & $D-\mathrm{H}$ & $\mathrm{H} \cdots A$ & $D \cdots A$ & $D-\mathrm{H} \cdots A$ \\
\hline $\mathrm{N} 1-\mathrm{H} 1 N 1 \cdots \mathrm{N} 4{ }^{\mathrm{i}}$ & $0.875(19)$ & $2.616(18)$ & $3.4230(14)$ & $153.8(15)$ \\
$\mathrm{N} 1-\mathrm{H} 2 N 1 \cdots \mathrm{O} 1^{\mathrm{ii}}$ & $0.882(18)$ & $2.533(19)$ & $3.3274(13)$ & $150.2(15)$ \\
$\mathrm{N} 2-\mathrm{H} 1 N 2 \cdots \mathrm{O} 2^{\mathrm{iii}}$ & $0.880(18)$ & $2.031(18)$ & $2.8866(12)$ & $163.7(16)$ \\
$\mathrm{C} 4-\mathrm{H} 4 A \cdots \mathrm{O} 1^{\text {ii }}$ & $0.944(16)$ & $2.460(16)$ & $3.2603(13)$ & $142.5(13)$ \\
\hline
\end{tabular}

Symmetry codes: (i) $-x+1, y+1 / 2,-z+5 / 2$; (ii) $x, y, z+1$; (iii) $-x,-y+1,-z+2$. 